\title{
Dimensionamento hidrológico para reservatório a fim de sanar as necessidades hídricas de Itabuna-BA
}

\section{Lucas Farias de Sousa1, Bruna Borges Soares ${ }^{2} \mathrm{e}$ Romário Oliveira de Santana ${ }^{3}$}

1 Estudante da Pós-graduação de Geotecnologias da Escola de Engenharia de Agrimensura. Engenheiro Ambiental formado pela Universidade Estadual do Sudoeste da Bahia, Brasil.

E-mail: eng.sousalucas@gmail.com 1 http://lattes.cnpq.br/6855382097221938 1 http://orcid.org/0000-0003-3152-2330

2 Mestre em Ciências Ambientais pela UESB. Engenheira Ambiental. Professora Assistente na Universidade Federal do Sul da Bahia, Brasil.

E-mail: bruna.soares@ufsb.edu.br http://lattes.cnpq.br/3842627585579289 (1) http://orcid.org/0000-0002-8259-6973

3 Doutorando no Programa de Desenvolvimento e Meio Ambiente na Universidade Estadual de Santa Cruz, Mestre em Ciências Ambientais e graduado em Engenharia Ambiental pela Universidade Estadual do Sudoeste da Bahia, Brasil.

E-mail: romarioambiental1@gmail.com 1 http://lattes.cnpq.br/1260225932521660 1 http://orcid.org/0000-0001-9603-0738

RESUMO: O presente estudo objetivou dimensionar um reservatório de regularização de vazão para atender as demandas no município de Itabuna - Bahia a partir de imagens de satélites. Para tal, foi estimada a população no ano de 2037 e sua demanda hídrica. A classificação supervisionada da carta do Landsat 8 de 2017 foi produzida para avaliação da área de estudo. 0 dimensionamento do reservatório foi realizado a partir da análise das curvas de nível. O mapa gerado a partir dessa análise foi sobreposto ao mapa de uso do solo para identificação e quantificação das classes alagadas. A população de Itabuna-BA está estimada para o ano de 2037 em 246.755 habitantes, para atender essa população o reservatório deve armazenar um volume de $53,29 \mathrm{hm}^{3}$ e altura de 14 metros, mantendo uma vazão de $1,33 \mathrm{~m}^{3} / \mathrm{s}$. A área alagada do reservatório foi de 1548 ha sendo que 1449 ha são áreas de pastagem e 99 ha de fragmentos florestais. O dimensionamento proposto, além de atender a demanda da população estimada, apresenta uma área de alagamento de $96,8 \%$ da cobertura vegetal, sendo esta composta por pastagem, desta forma o trecho é o mais recomendado para construção do reservatório.

Palavras-Chave: hidrologia, geoprocessamento, recursos hídricos

Hydrological Sizing for reservoir to attend the water needs of Itabuna-BA

ABSTRACT: The present study aimed to size a reservoir of water supply to attend the needs of Itabuna - Bahia from satellite images. So, was estimated a population by the year 2037 and its historical demand. Supervised classification of the Landsat 8 letter by 2017 was generated for evaluation of the study area. The sizing of the reservoir was carried out by analyzing the contour lines. The map generated from this analysis was superimposed on the landuse map for flooded classes identification and quantification. The estimated population in Itabuna-BA for the year 2037 was 246,755 inhabitants, to attend this population the reservoir should have a volume of $53.29 \mathrm{hm}^{3}$ and a height of 14 meters, maintaining a flow of $1.33 \mathrm{~m}^{3} / \mathrm{s}$. The reservoir wetland area was 1548 ha which 1449 ha are pasture areas and 99 ha are forest fragments. The proposed design attended the demand of the estimated population that presents an area of flooding $96.8 \%$ of the vegetation cover which is composed by pasture, it means that this stretch is the most recommended for the reservoir construction.

Keywords: hydrology, Geoprocessing, water resources 


\section{INTRODUÇÃO}

O crescimento populacional combinado com o aumento da atividade econômica, melhoria nos padrões de vida, urbanização e incertezas climáticas, têm aumentado a demanda mundial por água de boa qualidade em uma taxa superior à taxa de renovação associada ao ciclo hidrológico em diferentes partes do mundo (DOMENE; SAURI, 2006; HOUSE-PETERS; PRATT; CHANG, 2010; KENNEY et al., 2008; LEE; PARK; JEONG, 2012; HADDELAND et al., 2014; RATHNAYAKA et al., 2016). Acredita-se que até 2030, o mundo estará projetado para enfrentar um déficit global de água de $40 \%$ em relação ao cenário atual.

Em termos globais, é sabido que o Brasil possui grande oferta de água. Entretanto, este recurso natural encontra-se distribuído de maneira heterogênea no território nacional. Passam pelo território brasileiro em média cerca de $260.000 \mathrm{~m}^{3} / \mathrm{s}$ de água, porém $205.000 \mathrm{~m}^{3} / \mathrm{s}$ estão localizados na bacia do rio Amazonas, permanecendo para o restante do território $55.000 \mathrm{~m}^{3} / \mathrm{s}$ de vazão média, ou seja, há tanto escassez quanto abundância no território brasileiro (MELO, 2010; ANA, 2016).

No ano de 2016, o nordeste brasileiro apresentou um aumento na intensidade da seca em relação à registrada no ano de 2014. Os baixos índices de precipitação e a irregularidade do seu regime pluviométrico, aliados ao contexto hidrogeológico, contribuíram para a redução significativa da disponibilidade hídrica nesta região (ANA, 2016). Conforme informações da Base de Dados de Emergência do Painel Intergovernamental de Mudanças Climáticas (IPCC), aproximadamente 27 milhões de brasileiros foram atingidos pela seca no ano de 2014, totalizando um dano em torno de U\$ 5 biIhões (IPCC, 2017).

Como consequência, muitas cidades nordestinas vêm sofrendo com a diminuição da vazão dos rios e perda de qualidade de água. Dentre tais cidades, tem destaque a cidade de Itabuna-BA. O município é uma das cinco maiores cidades do Estado da Bahia, situada na Microrregião Homogênea Cacaueira e um importante polo de negócio da Costa do Cacau que concentra a maior parte da movimentação financeira de toda microrregião. $O$ desenvolvimento da cidade, expansão desordenada e a falta de políticas públicas, em especial nas nascentes situadas na bacia, bem como todos esses fatores aliados às mudanças climáticas, levaram a diminuição da disponibilidade hídrica no município.

Dados da Estação Fluviométrica de Ferradas (cod: 53170000) operada pela Agência Nacional das Águas (ANA), mostra que nos últimos 5 anos houve um déficit no balanço hídrico do Rio Cachoeira com valores de vazão abaixo da vazão média em muitos períodos nesses últimos anos. Itabuna possui dois outros pontos de captação de água que são em Rio do Braço e a Estação de Castelo Novo, localizado em um distrito de Ilhéus, entretanto, estas duas ficam um pouco longe do município ocasionando custos adicionais com distância de bombeamento de água, problemas com manutenção, perdas ao longo da linha de abastecimento e, segundo a ANA, não suprem a demanda que exige a cidade atualmente.

Ultimamente, estratégias de caráter emergenciais vêm sendo adotadas por algumas cidades que sofrem com os excessos nos períodos de chuva, carência nos períodos secos e precariedade do abastecimento para suprir a demanda de municípios, den- 
tre elas está o barramento para regularização de vazão. Este tipo de solução tem sido o principal instrumento na busca da sustentabilidade hídrica nas regiões onde os recursos hídricos são limitados ou apresentam uma distribuição temporal desfavorável. Esta medida pode ser uma alternativa viável para ajudar no melhor aproveitamento dos recursos hídricos podendo acumular os excessos de água que chegam na área de drenagem da bacia; aumentar a vazão para atender os períodos cujas vazões naturais são menores que as normais, podendo desativar alguma das outras estações de captação cortando custos, bem como minimizar as cheias que causam grandes prejuízos à população que vive às margens do rio $\mathrm{Ca}$ choeira.

Deste modo, a instalação de uma barragem na bacia do rio Cachoeira pode se mostrar uma alternativa eficaz evitando o desabastecimento sazonal, assim como evitar problemas de escassez hídrica semelhantes aos anos de 2015/16 ocorram em situações futuras.

Diante do exposto, este estudo teve como objetivo realizar o dimensionamento hidrológico de um reservatório para regularização de vazão com vista ao atendimento das necessidades hídricas do município de Itabuna-BA usando técnicas de geoprocessamento aliadas ao software SisCAH. Para tanto, foi estimada a demanda hídrica de Itabuna e Itapé, visto que a barragem proposta inclui alterações no corpo hídrico também da cidade vizinha a Itabuna - município de Itapé; foi também estimado o volume hídrico necessário para a reserva de água; e simulada a área física ideal ou aproximadamente ideal para o estabelecimento da barragem, levando em consideração questões específicas.
2 METODOLOGIA

\subsection{Delimitação e caracterização da área de estudo}

A bacia do rio Cachoeira (Figura 1) origina-se nas nascentes do rio Colônia, numa altitude de $800 \mathrm{~m}$, na Serra da Ouricana (município de Itororó), e atinge o seu patamar mais baixo na superfície litorânea do município de Ilhéus-BA. O rio Colônia, após estender-se por $100 \mathrm{~km}$, banhando os municípios de Itororó-BA, Itapetinga-BA e Itaju do Colônia-BA, tem sua confluência com o rio Salgado no município de Itapé-BA, passando então a receber o nome de rio Cachoeira (NACIF, 2000). O rio Salgado tem suas nascentes no município de Firmino Alves-BA e possui um curso de $64 \mathrm{~km}$ pelos municípios de Santa Cruz da Vitória-BA, Floresta Azul-BA, Ibicaraí-BA e Itapé-BA, onde desemboca no rio Colônia. O rio Cachoeira, após percorrer $50 \mathrm{~km}$ nos municípios de Itapé-BA, Itabuna-BA e llhéus-BA, tem a sua foz no local conhecido como Coroa Grande, ponto em que confunde as suas águas com as dos rios Santana e Fundão (CEPLAC, 1976; OLIVEIRA, 1997). 
Figura 1: Bacia Hidrográfica do Rio Cachoeira

Figure 1: Watershed Cachoeira River

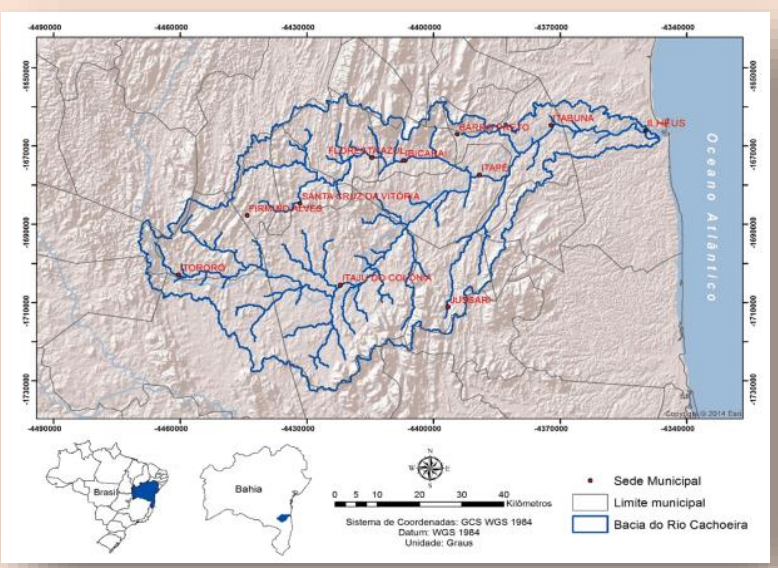

Segundo IBGE (2017), a população da Bacia do Rio Cachoeira é de aproximadamente 500.000 habitantes compreendendo as cidades de Itororó-BA, Firmino Alves-BA, Itaju do Colônia-BA, Santa Cruz da Vitória-BA, Floresta Azul-BA, Ibicaraí-BA, Itapé-BA, Barro Preto-BA, Itabuna-BA, Jussari-BA e Ilhéus-BA. Está limitada ao norte pelas bacias dos rios de Contas e Almada; ao sul, pelas bacias dos rios Pardo e Una; a oeste, pela bacia do rio Pardo; e a leste, pelo Oceano Atlântico.

A qualidade das águas do Cachoeira está diretamente relacionada às características naturais, à ocupação e aos usos da terra de sua bacia. Além das práticas agropecuárias inadequadas, desmatamentos e atividades urbanas e industriais contribuem para a degradação dos seus recursos hídricos (SCHIAVETTI; SCHILLING; OLIVEIRA, 2005).

A região de llhéus apresenta estações chuvosas no período de março a julho e estações um pouco mais carente de chuvas nos demais meses. Em Itabuna-BA, Itapé$B A$, porção centro-oeste da bacia, Itaju do Colônia-BA, Itororó-BA e Itapetinga-BA e proximidades, o período chuvoso acontece nos meses de novembro - dezembro e março - abril. A rede hidrometeorológica da bacia do rio Cachoeira contempla 09 estações fluviométricas e 13 estações pluviométricas.

\subsection{Material e Métodos}

Foram analisados os trechos que, visualmente, ao dimensionar a barragem teria a menor probabilidade de deslocamento de moradores, pouca supressão de mata fechada e longe de rodovias importantes como é o caso da BA 120. O trecho escolhido foi no rio Colônia entre os municípios de Itapé-BA e Itaju do Colônia-BA onde a vazão por parte do afluente é maior que no rio Salgado, o deslocamento de pessoas seria menor, bem como a supressão de mata fechada e intervenção de rodovia. Consequentemente, devido a escolha do lugar para o barramento ser antes das cidades de Itabuna-BA e Itapé-BA, foi necessário o dimensionamento da demanda hídrica para ambas as cidades.

A descrição da estimativa de demanda hídrica, dados hidrológicos, softwares empregados e o dimensionamento da barragem conformam o escopo das seções subsequentes.

\subsubsection{Estimativa da demanda hídrica nos municípios de Itabuna e Itapé}

As informações demográficas foram coletadas por meio da base de dados do site do Superintendência de Estudos Econômicos e Sociais da Bahia (SEI). Então, foi feita a estimativa da população no ano de 2037 usando a metodologia proposta pelo Manual de Procedimentos Técnicos e Administrativos de Outorga de Direito de Uso de 
Recursos Hídricos (AGÊNCIA NACIONAL DE ÁGUAS, 2013).

Após a estimativa da população nos municípios, calculou-se a demanda hídrica da população no ano de 2037 conforme a equação (1) e por meio do Plano Estadual de Recursos Hídricos (BAHIA, 2001) efetivou-se a quantidade estipulada de demanda consultiva na Bacia do Leste e transportou para a realidade da Bacia do Rio Cachoeira, calculando as porcentagens de demanda hídrica para população urbana, população rural, rebanho, indústria e irrigação.

$V c=\left(\frac{P r \times q_{p c}}{1000}\right) \times K_{1} \times K_{2}$

Onde:

$V c$ = Vazão de cálculo;

$q_{p c}=$ Quota per capita;

$\operatorname{Pr}=$ População no ano projetado;

$K_{1}=$ Coeficiente de maior consumo diário;

$K_{2}=$ Coeficiente de maior consumo horário;

\subsubsection{Dados hidrológicos}

Os dados fluviométricos empregados no presente estudo foram obtidos a partir do Sistema de Informações Hidrológicas da Agência Nacional de Águas (ANA). Os dados de evaporação foram apropriados a partir do Instituto Nacional de Meteorologia (INMET), obtidos pela estação meteorológica de llhéus.

Para a condução do estudo, primeiramente foram escolhidas estações fluviométricas em operação na área de drenagem da bacia hidrográfica do rio Cachoeira. A extensão da série histórica e a disponibilidade de dados de vazão constituíram os critérios empregados para a seleção da estação flu- viométrica. 0 método de dimensionamento empregado neste trabalho demanda vazões médias mensais e totais diários evaporados. Nas situações em que as séries históricas selecionadas apresentaram falhas, efetivouse o pré-processamento excluindo todos os meses com falhas, deste modo, trabalhando com dados mais consistidos.

\subsubsection{Sistema Computacional para Análises Hidrológicas (SisCAH)}

Para a manipulação dos dados hidrológicos foi empregado o SisCAH, software de domínio público desenvolvido e disponibilizado pelo Grupo de Pesquisa em Recursos Hídricos da Universidade Federal de Viçosa. O software foi utilizado para a condução das seguintes tarefas:

- Pré-processamentos das séries históricas de vazão, com subsequente seleção dos períodos empregados para o dimensionamento do reservatório;

- Determinação, por estação fluviométrica, das vazões médias de longo período. Segundo Tucci (2004), a vazão média de longo período ou vazão média de longa duração pode ser definida como a média das vazões da série de dados disponíveis e corresponde à máxima vazão possível a ser regularizada em qualquer curso d'água, abstraindo-se as perdas;

- Determinação da vazão a ser regularizada.

\subsubsection{Dados fisiográficos}

A partir do Sistema de Informações Hidrológicas da ANA, foi obtido o arquivo no formato shapefile da Bacia Atlântico Trecho Leste. Este shapefile foi utilizado para a determinação da localização geográfica das 
estações hidrológicas e da rede de drenagem da bacia do Rio Cachoeira. Os modelos obtidos na ANA estavam em Sistemas de Coordenadas Geográficas América do Sul 1969 e Datum América do Sul 1969 sendo transformados para Coordenadas Geográficas SIRGAS 2000 UTM Zona 24s e Datum SIRGAS 2000 para melhor adequação aos requisitos brasileiros.

Adicionalmente, foram empregados shapes SRTM (Shuttle Radar Topography Mission) para a determinação da relação entre o volume reservado e a área do espelho d'água de cada cota estipulada para o dimensionamento do reservatório. Estes shapes foram obtidos a partir do monitoramento por satélite da NASA pelo Earth Explorer com dados de resolução espacial de $30 \mathrm{~m}$ e 32 bits os quais são disponíveis gratuitamente em seu site.

Para a confeç̧ão do mapa de uso e ocupação do solo foi adquirido uma carta do Landsat 8 do ano de 2017 pelo site do INPE o qual foram feitas classificações a fim de identificar os principais usos da terra na área de drenagem da bacia classificando-as em: Fragmentos Florestais, Pastagem Se$\mathrm{ca} /$ Solo exposto, Pastagem verde, Área de Influência Urbana.

Todos os procedimentos que envolveram o tratamento e manipulação de shapefiles e imagens de satélite foram realizados com o auxílio do software ArcGIS 10.1, desenvolvido pelo Environmental Systems Research Institute (ESRI).

\subsubsection{Método empregado para o dimensio- namento do volume do reservatório}

Ao analisar as estações fluviométricas contidas na área de drenagem da bacia foi estabelecido que dimensionar a barragem próxima à estação da Estiva De Baixo seria a melhor opção por obter uma maior vazão de longa duração, menor probabilidade de interdição de rodovias importantes e deslocamento de população. Nesse contexto, o software ArcGIS 10.1 permitiu calcular, a partir da análise das curvas de nível no entorno próximo à estação fluviométrica, diferentes volumes de reservação e áreas do espelho d'água correspondente.

Nessa etapa do trabalho, as cartas no modelo SRTM foram manipuladas pelo programa ArcGIS 10.1 e foram unidas em uma única imagem utilizando-se a ferramenta Mosaic to New Raster. As imagens obtidas do Earth Explorer estão em coordenadas geográficas sobre o Datum planimétrico WGS1984. No entanto, para estudos sobre bacias hidrográficas recomenda-se o uso de uma projeção cartográfica equivalente, ou seja, aquela que mantém as distorções das áreas proporcionais (FERREIRA; MOURA; CASTRO, 2012). Dessa forma, aplicou-se a projeção SIRGAS 2000 UTM Zona 24S sobre o mosaico utilizando-se a ferramenta Project Raster afim de alterar o seu sistema de coordenadas. Adicionalmente, utilizou-se a ferramenta Fill, com o objetivo de se eliminar pequenas imperfeições, como depressões e picos, no modelo digital. Posteriormente, foi delimitada a bacia do Rio Cachoeira através das ferramentas Flow Direction, Flow Accumulation, e Watershed todas estas ferramentas contidas na extenção $\mathrm{Hi}$ drology do ArcGIS 10.1. Em seguida, com o raster já ajustado e com a delimitação da bacia, utilizou-se a ferramenta Contour (Spatial Analyst) para a criação de curvas de nível à princípio com intervalo de cinco metros de espaçamento e depois com intervalos de 2 metros, totalizando em 7 níveis de barramento. 
A partir da cota de instalação do barramento, assumiu-se um aumento da soleira da barragem, com o objetivo de se apropriar a área de espelho d'água e seu respectivo volume. Nesta etapa de manipulação do MDE foi necessária a criação de polígonos. Cada polígono correlacionou uma altura distinta da barragem com uma área superficial de alagamento. Para o cálculo do volume de cada polígono gerado foi necessária a interpolação das curvas de nível, criando uma estrutura de grade triangular (TIN) que representa uma superfície por meio de conjuntos de faces triangulares. A partir disso, utilizou-se a ferramenta Polygon Volume para se determinar a área e o volume correspondente.

\subsubsection{Quantificação da área alagada}

A quantificação da perda de habitat devido a área alagada será feita a partir do mapa de uso da terra sobreposto ao mapa gerado da área alagada, deste modo, quantificando a quantidade de classe tomada pela água.

Os dados de uso e ocupação do solo da BHRC foram obtidos a partir da manipulação de imagens de satélite LANDSAT 8 TM registradas para a área em estudo no ano de 2017, respectivamente, da órbita 216/70 e com resolução espacial de $30 \mathrm{~m} \times 30 \mathrm{~m}$, compatível com a escala de trabalho de $1: 100.000$. Para a manipulação das imagens, incluindo processos de composição de bandas, correções atmosféricas e geométricas, foi utilizado o software ArcGIS 10.1. Preparada a imagem iniciou-se o processo de classificação supervisionada tendo em vista a caracterização das assinaturas multiespectrais das diferentes tipologias de uso e ocupação do solo, também utilizando o módulo disponível no ArcGIS 10.1. A amostra representativa de cada uma das diferentes tipologias de uso e ocupação refere-se a uma determinada quantidade de pixels coletada na imagem e que serve de parâmetro para classificação. Dessa forma é possível captar a informação existente na amostra e expandir a sua ocorrência por toda a imagem a partir da adoção de modelos de distribuição estatística multivariada, sendo neste caso utilizado o da máxima verossimiIhança. Após a classificação aplicou-se o processo de filtragem estatística 3 × 3 majoritária no sentido de suavizar a ocorrência de pixels isolados em pequenas áreas dentro de classes de maior extensão areal.

\section{RESULTADOS E DISCUSSÃO}

A estimativa da população das cidades de Itabuna-BA e Itapé-BA para 2037 é de 246.755 habitantes, totalizando uma demanda hídrica para o ano de 2037 de 1,273 $\mathrm{m}^{3} / \mathrm{s}$ para as respectivas cidades. Utilizando-se o software SisCAH, foi encontrado o valor da vazão média de longo período da estação 53140000, localizada próximo à Estiva de Baixo, cujo valor foi de $8,739 \mathrm{~m}^{3} / \mathrm{s}$. A partir da curva de permanência da estação fluviométrica, também estabelecida com auxílio do software SisCAH, foi apropriada a vazão $Q_{90}$ da estação fluviométrica igual a $117,8 \mathrm{~L} / \mathrm{s}$.

O dimensionamento hidrológico do reservatório foi feito por tentativas, a partir da construção das curvas de nível, que representa a variação do volume máximo o qual cada cota pode armazenar. A altura máxima da barragem foi avaliada em função da topografia no ponto do barramento, o qual permitiu a implantação de uma barragem com até $14 \mathrm{~m}$ de altura total, atin- 
gindo a cota $128 \mathrm{~m}$ considerando uma lâmina máxima de sangria de $1,00 \mathrm{~m}$ e uma borda livre de 1,0 $\mathrm{m}$. O volume útil máximo da barragem seria o correspondente à cota 126 m (Figura 2), para uma lâmina d'água máxima de $12,0 \mathrm{~m}$. No Quadro 1, encontram-se os dados de cota, área e volume obtidos a partir do MDE.

Figura 2: Área inundada na Cota $126 \mathrm{~m}$

Figure 2: Flood area in $126 \mathrm{~m}$ Contour

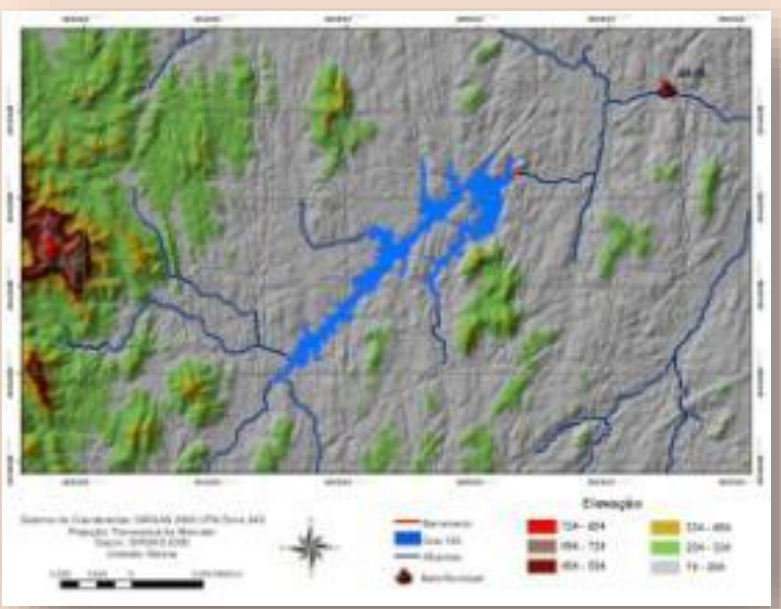

Quadro 1: Relação cota $x$ área x volume

Frame 1: Contour $x$ área $x$ volume relation

\begin{tabular}{|c|c|c|}
\hline Cota $(\mathbf{m})$ & Área $\left.\mathbf{( k m}^{\mathbf{2}}\right)$ & Volume $\mathbf{( h m}^{\mathbf{3}} \mathbf{)}$ \\
\hline 114 & - & - \\
\hline 116 & 0,17 & 0,34 \\
\hline 118 & 0,48 & 1,14 \\
\hline 120 & 1,37 & 3,5 \\
\hline 122 & 1,9 & 6,9 \\
\hline 124 & 6,3 & 17,9 \\
\hline 126 & 11,9 & 39,1 \\
\hline 128 & 17,16 & 70,4 \\
\hline
\end{tabular}

As simulações foram feitas considerando a capacidade de armazenamento desde a cota $116\left(0,34 \mathrm{hm}^{3}\right)$, até a cota $128(70,4$ $\mathrm{hm}^{3}$ ), variando de 2 em $2 \mathrm{~m}$. À cada simulação feita no ArcGIS 10.1 procedeu-se, simultaneamente, os cálculos para regularização de vazão com área alagada, evaporação diária e vazão como dados de entrada no
SisCAH. Para cada capacidade de acumulação foi determinada a vazão regularizada por tentativas, buscando-se o valor demandado pelas duas cidades para o qual o nível de atendimento informado pelo $\mathrm{Sis} C \mathrm{AH}$ fosse o mais próximo possível de $1,272 \mathrm{~m}^{3} / \mathrm{s}$. 0 resultado das simulações está resumido no Quadro 2. Considerou-se como volume mínimo operacional o armazenado na cota $124 \mathrm{~m}(10,0 \mathrm{~m}$ em relação à base da barragem), aproximadamente $17,9 \mathrm{hm}^{3}$.

Quadro 2: Vazões regularizadas.

Frame 2: Regulated outflows.

\begin{tabular}{|c|c|c|}
\hline Cota (m) & Capacidade $\left(\mathbf{h m}^{\mathbf{3}}\right)$ & Qreg $\left(\mathbf{m}^{\mathbf{3}} \mathbf{s} \mathbf{~}\right)$ \\
\hline 114 & - & $*$ \\
\hline 116 & 0,34 & $*$ \\
\hline 118 & 1,14 & $*$ \\
\hline 120 & 3,5 & $*$ \\
\hline 122 & 6,9 & $*$ \\
\hline 124 & 17,9 & 0,71 \\
\hline 126 & 39,1 & 1,36 \\
\hline 128 & 70,4 & 2,19 \\
\hline
\end{tabular}

* Cotas impossibilitadas de se obter vazão regularizada para o corpo hídrico.

Na quantificação da vegetação perdida pela área alagada, como mostra a Figura 3, constatou-se que será coberto cerca de 1449 ha de pastagem em toda a sua extensão e 99 ha de fragmentos florestais. Ressalva-se que na área do barramento não existe qualquer infraestrutura pública ou privada que possa ser atingida diretamente com a estrutura da barragem de acordo à classificação da imagem. Sendo assim, tendo menores impactos ambientais, já que a área alagada será predominantemente de pastagem, bem como menores custos com desapropriação de terras, pois as áreas de pasto são de pequeno - médio produtores rurais. 
Figura 3: Mapa de uso do solo da região afetada pelo barramento

Figure 3: Landuse map of the region affected by the reservoir

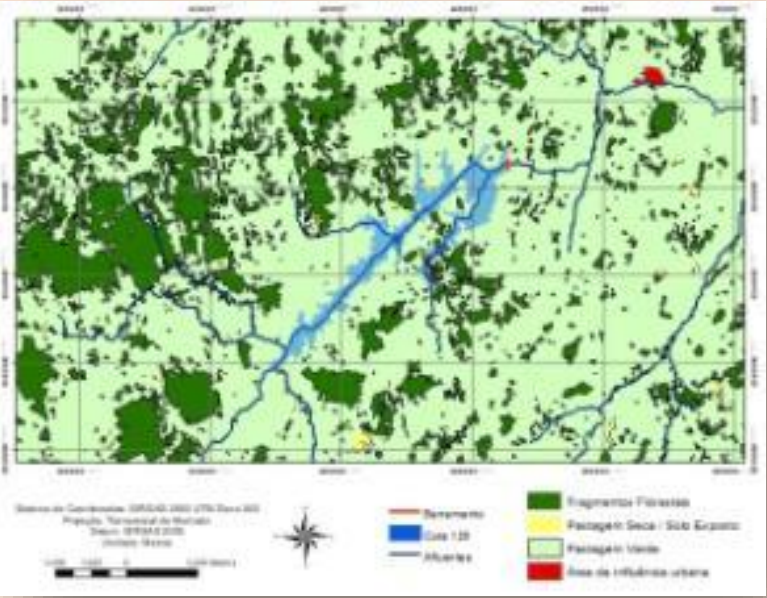

\section{CONCLUSÕES}

Verificou-se, do ponto de vista hidrológico, que o barramento mais indicado para o abastecimento de uma população futura de 246.755 habitantes possua as seguintes características: capacidade de armazenamento de um volume de $53,29 \mathrm{hm}^{3}$; altura de 14 metros considerando $1 \mathrm{~m}$ de lâmina d'água e o mesmo valor de folga sob o rio Colônia; além de manter uma vazão de $1,33 \mathrm{~m}^{3} / \mathrm{s}$.

$\mathrm{A}$ área alagada pelo reservatório corresponde a 1548 ha, sendo que $96,8 \%$ deste total corresponde a pastagem como cobertura vegetal e 3,2\% corresponde a fragmentos florestais. A área alagada também não alcança nenhuma área de influência urbana, pois o local não apresenta presença de infraestrutura pública, além da estrada estadual BA-120, bem como não há vila ou povoado na área a ser alagada.

O reservatório proposto para regularização da disponibilidade hídrica no município de Itabuna sendo construído no trecho identificado, além de suprimir as demandas da população, não deve causar grandes impactos ecológicos.

Como recomendações do presente trabalho sugere-se replicar a metodologia de avaliação de volumes de reserva usados no presente artigo para bacia hidrográfica que apresente modelo digital do terreno estabelecido com maior precisão. Mesmo que no presente estudo a ferramenta Fill, disponível no software ArcGIS 10.1, tenha sido empregada para correção de depressões espúrias, sua aplicação sem a adequada verificação de campo pode produzir a eliminação tanto dos erros de altitude quanto as depressões reais existentes, produzindo modelos que não se apresentam confiáveis para a descrição da morfologia da bacia hidrográfica.

\section{REFERÊNCIAS}

AGÊNCIA NACIONAL DE ÁGUAS (BRASIL). Conjunturas dos Recursos Hídricos do Brasil. Brasília, 2016. Disponível em: <http:// www3.snirh.gov.br/portal/snirh/centrais-de -conteudos/conjuntura-dos-recursos-hidri cos/informe-conjuntura-2016.pdf > Acesso em: 17 jan. 2018.

AGÊNCIA NACIONAL DE ÁGUAS (BRASIL). Manual de procedimentos técnicos e administrativos de outorga de direito de uso de recursos hídricos 2013, Brasília: 2013. Disponível em: <http://arquivos.ana.gov.br/ institucional/sof/MANUALDEProcedimentos TecnicoseAdministrativosdeOUTORGAdeDireitodeUsodeRecursosHidricosdaANA.pdf>.

Acesso em: 30 jun. 2018

BAHIA. SECRETARIA DE RECURSOS HÍDRICOS - SRH. Programa de recuperação das bacias hidrográficas dos Rios Cachoeira e Almada. Diagnóstico Regional. Caracteri- 
zação Hidrológica. Ilhéus - BA, SRH/UESC, v. 1, Tomo IV. 2001

COMISSĀO EXECUTIVA DO PLANO DA LAVOURA CACAUEIRA - CEPLAC. Recursos Hídricos. Rio de Janeiro: IICA/ CEPLAC, 1976. 280p.

DOMENE, E; SAURÍ, D. Urbanization and water consumption: Influencing factors in the metropolitan region of Barcelona. Urban Studies, Barcelona, v. 43, n. 9, p. 16051623, ago. 2006.

FERREIRA, R. G.; MOURA, M. C. O.; CASTRO, F. S. Caracterização morfométrica da subbacia do Ribeirão Panquinhas, ES. Enciclopédia Biosfera, Rio Verde, v.8, n.15, p.22472256, out. 2012.

HADDELAND, I et al. Global water resources affected by human interventions and climate change. Proceedings of the. National Academy of Sciences. USA, 4 mar 2014, 111 (9), 3251-3256. https://doi.org/10.1073/ pnas. 1222475110

HOUSE-PETERS, L., PRATT, B., CHANG, H. Effects of urban spatial structure, sociodemographics and climate on residential water consumption in Hillsboro, Oregon. J. Am. Water Resour. Assoc. 46 (3), 461-472, 7 jun 2010. https://doi.org/10.1111/i.17521688.2009.00415.x

INSTITUTO BRASILEIRO DE GEOGRAFIA E ESTATÍSTICA - IBGE (2017). Disponível em: <http://www.cidades.ibge.gov.br/v3/cida des/home-cidades $>$. Acesso em: 03 fev. 2018.

INTERGOVERNMENTAL PANEL ON CLIMATE CHANGE - IPCC. Base de Dados de Emergência de Desastres, 2017. Disponível em: $<$ http://www.emdat.be/disaster profiles/in dex.html>. Acesso em: 20 dez 2017.

KENNEY, D.S.et al. Residential water demand management: lessons from Aurora, Colorado. Journal of The American Water
Resources Association, 10 jan 2008, v 44, n 1, 192-207. https://doi.org/10.1111/j.17521688.2007.00147.x

LEE, D., PARK, N., JEONG, W. End-use analysis of household water by metering: the case study in Korea. Water Environmental Journal. South Korea, 11 jan 2012, v. 26, n. 4, 455-464. https://doi.org/10.1111/i.17476593.2011.00304.x

MELO, C. R. Análise do eixo leste da transposição do Rio São Francisco face aos cenários de uso previstos. 2010. Dissertação (Mestrado) - UFPE. Programa de PósGraduação em Engenharia Civil, 2010.

NACIF, P. G. S. Bacia hidrográfica do rio Cachoeira: dinâmica do meio físico com ênfase aos domínios pedológicos.Tese (Doutorado em Agronomia) - Universidade Federal de Viçosa, Viçosa (MG), 2000.

OLIVEIRA, M. C. R. As relações ambientais da Bacia Hidrográfica do Rio Cachoeira (sul da Bahia). Trabalho de Conclusão de Curso, Ilhéus: Editus, 1997.

RATHNAYAKA, K., et al., Prediction of urban residential end-use water demands by integrating known and unknown water demand drivers at multiple scales I: Model development. Resources, Conservation and Recycling, fev. 2016, v. 117, parte B, p. 85-92.

SCHIAVETTI, A.; SCHILLING, A. C.; OLIVEIRA, HT de. Caracterização sócio-ambiental da bacia hidrográfica do Rio Cachoeira, Sul da Bahia, Brasil. Conceitos de Bacias Hidrográficas: Teoria e Aplicações. Editus, Ilhéus, p. 141-161, 2002.

TUCCI, C. E. M. Hidrologia - Ciência e Aplicação. 3a Ed. Porto Alegre: ABRH, 2004. 943 p.

USGS, Earth Eplorer. Disponível em: <https: //earthexplorer.usgs.gov/>. Acesso em: 28 nov. 2017. 


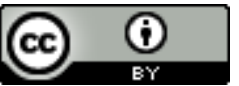

License information: This is an openaccess article distributed under the terms of the Creative Commons Attribution License, which permits unrestricted use, distribution, and reproduction in any medium, provided the original work is properly cited.

Artigo recebido em 27 de março de 2018.

Avaliado em 30 de junho de 2018.

Aceito em 02 de julho de 2018.

Publicado em 28 de fevereiro de 2019.

\section{Como citar este artigo (ABNT):}

SOUSA, Lucas Farias de; SOARES, Bruna Borges; SANTANA, Romário Oliveira de. Dimensionamento hidrológico para reservatório a fim de sanar as necessidades hídricas de Itabuna-BA. Estação Científica (UNIFAP), Macapá, v. 8, n. 2, p. 69-79, maio/ago. 2018. 